DySPhagia

\title{
Dysphagia screening in subacute care settings using the Italian version of the Royal Brisbane and Women's Hospital (I-RBWH) dysphagia screening tool
}

\author{
Screening della disfagia nelle unità di cure sub-acute utilizzando la versione \\ italiana del Royal Brisbane and Women's Hospital (I-RBWH) dysphagia screening \\ tool \\ F. MOZZANICA ${ }^{1}$, L. SCARPONI ${ }^{1}$, S. PEDRALI ${ }^{1}$, N. PIZZORNI ${ }^{2}$, C. PINOTTI ${ }^{3}$, F. FOIENI ${ }^{3}$, G. ZUCCOTTI ${ }^{4}$, \\ A. SCHINDLER ${ }^{1}$ \\ ${ }^{1}$ Phoniatric Unit, Department of Biomedical and Clinical Science, L. Sacco Hospital University of Milan, Milan, Italy; \\ ${ }^{2}$ Department of Rehabilitation Medicine, Casa di Cura Privata del Policlinico, Milan, Italy; ${ }^{3}$ SubAcute Care Unit, \\ L. Sacco Hospital, Milan, Italy; ${ }^{4}$ Pediatric Unit, Department of Biomedical and Clinical Science, Buzzi Hospital \\ University of Milan, Milan, Italy
}

\section{SUMMARY}

The large majority of the available dysphagia screening tools has been developed for the stroke population. Only few screening tools are suitable for heterogeneous groups of patients admitted to a subacute care unit. The Royal Brisbane and Women's Hospital (RBWH) dysphagia screening tool is a nurse-administered, evidence-based swallow screening tool for generic acute hospital use that demonstrates excellent sensitivity and specificity. No Italian version of this tool is available to date. The aim of this study was to determine the reliability and screening accuracy of the Italian version of the RBWH (I-RBWH) dysphagia screening tool. A total of 105 patients consecutively admitted to a subacute care unit were enrolled. Using the I-RBWH tool, each patient was evaluated twice by trained nurses and once by a speech and language pathologist (SLP) blind to nurses' scores. The SLP also performed standardised clinical assessment of swallowing using the Mann assessment of swallowing ability (MASA). During the first and the second administration of the I-RBWH by nurses, 28 and 27 patients, respectively, were considered at risk of dysphagia, and 27 were considered at risk after SLP assessment. Intra- and inter-rater reliability was satisfactory. Comparison between nurse I-RBWH scores and MASA examination demonstrated a sensitivity and specificity of the I-RBWH dysphagia screening tool up to $93 \%$ and $96 \%$, respectively; the positive and negative predictive values were $90 \%$ and $97 \%$, respectively. Thus, the current findings support the reliability and accuracy of the I-RBWH tool for dysphagia screening of patients in subacute settings. Its application in clinical practice is recommended.

KEY WORDS: RBWH, Dysphagia • Screening • Subacute care unit

\section{RIASSUNTO}

La maggior parte dei test di screening per la disfagia è stato sviluppato per essere utilizzato in pazienti con stroke. Solo pochi strumenti risultano applicabili a popolazioni più eterogenee di pazienti, come quelli ricoverati nelle unità di cure per Sub-acuti. Tra questi, il Royal Brisbane and Women's Hospital (RBWH) dysphagia screening tool è stato concepito per essere utilizzato da personale infermieristico e possiede un'eccellente sensibilità e specificità. Al momento non è disponibile una versione italiana di questo strumento. Scopo del lavoro è di analizzare l'affidabilità e l'accuratezza nello screening della versione italiana del RBWH(I-RBWH) dysphagia screening tool. A tal fine sono stati arruolati 105 pazienti, tutti ricoverati presso l'unità di cure Sub-acute. Ogni paziente è stato valutato con il I-RBWH dysphagia screening tool da personale infermieristico (per due volte) e da una logopedista. Quest'ultima, non solo era all'oscuro dei risultati ottenuti durante la valutazione infermieristica, ma ha anche eseguito una valutazione standardizzata delle abilità deglutitorie utilizzando il Mann Assessment of Swallowing ability (MASA). L'affidabilità intra- e inter-rater si sono rilevate soddisfacenti. Il confronto tra i risultati ottenuti dal personale infermieristico durante la somministrazione del I-RBWH e i punteggi del MASA hanno dimostrato un'eccellente sensibilità (93\%), specificità (96\%), valore predittivo positivo (90\%) e valore predittivo negativo (97\%). Questi dati supportano l'affidabilità e l'accuratezza del I-RBWH dysphagia screening tool nello screening della disfagia nei pazienti ricoverati nelle unità di cure per Sub-acuti. Il suo utilizzo in clinica è pertanto raccomandabile.

PAROLE CHIAVE: RBWH • Disfagia $\bullet$ Screening $\bullet$ Unità di cure per sub acuti 


\section{Introduction}

The estimated incidence of dysphagia in hospitalised patients ranges between $15 \%$ and $30 \%{ }^{12}$. These findings are not surprising since the prevalence of dysphagia is high in common diseases - it exceeds $50 \%$ in stroke patients, is present in 10-30\% of individuals older than 65 years ${ }^{3}$ and may be as high as $84 \%$ in patients with Parkinson's disease ${ }^{4}$. Dysphagia limits the safe ingestion of adequate amounts of food and liquids thus placing the patient at increased risk for poor nutrition, dehydration, aspiration pneumonia and morbidity in general ${ }^{5}$. In addition, patients affected by dysphagia experience an increased risk of hospital admission, delayed discharge and dependence on health services (e.g. increased nursing time and physician consultations) thereby increasing health care costs ${ }^{6}$. For these reasons, early identification of dysphagia is mandatory since it can reduce the incidence of clinical complications and may improve outcomes in these patients ${ }^{7-9}$. Even if clinical and instrumental assessment using either videofluoroscopic swallowing study (VFSS) or fibre optic endoscopic evaluation of swallowing (FEES) is considered the "gold standard" for identification of swallowing dysfunctions ${ }^{10-12}$, the approach has some limitations. Instrumental assessment is usually not available on a 24-hour basis and not all patients can be carried to the radiology department and correctly positioned, even with specially adapted chairs ${ }^{13}{ }^{14}$. As a consequence, it appears difficult to assure a well-timed screening of dysphagia, especially outside standard working hours on weekdays. Moreover, instrumental assessment is usually not available at patient admission. In order to take decisions on feeding for newly admitted patients and to reduce the number of patients requiring VFSS or FEES and prevent dysphagia related complications, several dysphagia screening tools have been proposed ${ }^{215-23}$.

The large majority of dysphagia screening tools have been developed for the stroke population and consequently cannot be readily used for a more heterogeneous population, such as that usually found in acute care settings, where more than half of the population presents a swallowing disorder due to a disease different from stroke ${ }^{24}$. Only few screening tools for patients with different diagnoses are available 1720212325 . Unfortunately, these latter are too time-consuming to be considered acceptable for a screening assessment where the cost of the test and the time taken to administer it is fundamental ${ }^{226}$. The screening tool developed by Logemann et al. ${ }^{17}$, in fact, is rather long as it includes 28 items in 5 categories: (1) 4 items on medical history variables; (2) 6 items on behavioural variables; (3) 2 items on gross motor variables; (4) 9 items on observations from oromotor testing; and (5) 7 items on observations during trial swallows. On the other hand, the screening tool developed by Stewart ${ }^{20}$ focuses only on patients with intellectual disabilities. The volume-viscos- ity swallow test for clinical screening of dysphagia and aspiration developed by Rofes et al. ${ }^{23}$ appears to be too time-consuming and complex; finally, in the Yale Swallow protocol developed by Suiter et al. ${ }^{25}$ no exclusion criteria, with the exception of the absence of tracheostomy tube, were established. Therefore, while it is applicable to large population, a triaging approach is needed to reduce the number of patients who are screened by a water swallow test.

To overcome these difficulties, the Royal Brisbane and Women Hospital (RBWH) dysphagia screening tool has recently been developed ${ }^{2}$. This latter is a nurse-administered, evidence-based swallow screening tool for generic acute hospital use based on the triaging concept. It consists of three steps: 1. a two-phase question screen; 2. a water swallow test, as appropriate; 3 . a swallowing management plan. The two-phase question screen reflects the perception that identification of 'at-risk' patients should come from a combination of (1) previous medical history/ records and (2) specific clinical indicators. Phase 1 of the screening tool uses evidence-based medical diagnoses known to have a high association with dysphagia and risk for aspiration, such as stroke, neurological involvement, head injury, or head and neck surgery ${ }^{1827}$. A negative indicator for any of the medical diagnoses allows the patient to start a general diet with liquids, while a positive indicator prompts the nurse to complete phase 2 . Phase 2 gathers from the patient's (or their family/carer's) information specifically related to feeding and swallowing. If any of the phase 2 dysphagia indicators are present, the patient is placed nil by mouth (NBM) and referred for formal dysphagia assessment. Individuals without phase 2 dysphagia indicators proceed to step 2 and are administered a water swallow test with $90 \mathrm{ml}$ of water. The nurse is prompted to observe for (1) coughing during or between swallows or up to one minute after swallowing, (2) wet or "gurgly" voice quality post-swallow and (3) increased respiratory rate post-swallow ${ }^{216}$. Depending on the results of the screening evaluation, patients with a positive result in the dysphagia screening examination are referred to a SLP.

In the original study by Cichero et al. ${ }^{2}$, the RBHW dysphagia screening tool demonstrated a sensitivity of $95 \%$, a specificity of $97 \%$, a positive predictive value of $92 \%$ and a negative predictive value of $98 \%$ compared to formal clinical assessment. Nurse compliance with administering the tool on each new admission was $83 \%$. In addition, the authors speculated that the use of the RBHW dysphagia screening tool may improve the quality of care and save healthcare costs. However, one of the major limitations of the original study lies in the lack of blinding, and consequently the interpretation of the screening results needs caution.

The aim of this study was to determine the reliability and screening accuracy of the Italian version of the Royal Brisbane and Women's Hospital (I-RBWH) dysphagia screen- 
ing tool using a blinded format between nurse screening and clinician assessment. The underlying hypothesis is that the I-RBWH is both reliable and accurate for screening dysphagia in subacute care settings. The importance of the study lies in the need for a simple, quick and accurate dysphagia screening tool to be applied in everyday clinical practice for early recognition and management of manage dysphagia in a heterogeneous population.

\section{Materials and methods}

The study consisted of 4 phases: item generation (phase 1), nurse training (phase 2), reliability analysis (phase 3) and screening accuracy analysis (phase 4). All data were collected prospectively and each subject enrolled in the study provided written informed consent. Only patients admitted to the Subacute Care Unit of our hospital in the period between 1 May 2015 and 30 September 2015 were included. The Subacute Care Unit is a comprehensive inpatient care unit, designed for patients who have an acute illness, injury, or exacerbation of a disease process; it is a goal oriented treatment rendered immediately after, or instead of, acute hospitalisation to treat one or more specific active complex medical conditions or to administer one or more technically complex treatments, in the context of a person's underlying long-term condition and overall situation.

The study was carried out according to the Declaration of Helsinki. The study design was approved by the Institutional Review Boards of our hospital.

\section{I-RBWH item generation (phase 1)}

Cross-cultural adaptation of the RBWH dysphagia screening tool was performed using standard techniques ${ }^{28} 29$; the authors of the RBWH were contacted and permission was obtained to start the project. Items of the original RBWH dysphagia screening tool were translated into Italian by one professional translator and two bilingual investigators. Two independent phoneticians familiar with the process of instrument validation examined semantic, idiomatic and conceptual issues, and further refined these versions. A final consensus version was obtained and given to two professional translators to produce a literal translation into English. The two translators and an expert committee synthesised the results of the translations in an English back translated version that was compared with the original one to check that they had the same semantic value in order to obtain the final version of the I-RBWH dysphagia screening tool.

\section{Nurse training (phase 2)}

Similar to the study of Cichero et al. ${ }^{2}$ the training package consisted of a 30-minute presentation prepared by the hospital speech pathology department and carried out by one SLP. During training, information regarding anatomy
Table I. Aetiological factors leading to subacute care unit admission in the cohort of patients $(n=105)$.

\begin{tabular}{lcccc} 
& Number & Percentage & \multicolumn{2}{c}{ Sex } \\
& & & M & F \\
$\begin{array}{l}\text { Clinical consequences of } \\
\text { infectious diseases }\end{array}$ & 32 & $30 \%$ & 16 & 16 \\
$\begin{array}{l}\text { Complications of surgical or } \\
\text { medical treatments }\end{array}$ & 26 & $25 \%$ & 11 & 15 \\
Cardiac failure & 21 & $20 \%$ & 11 & 10 \\
Diabetes mellitus & 9 & $8 \%$ & 3 & 6 \\
Other & 17 & $16 \%$ & 8 & 9 \\
\hline
\end{tabular}

and physiology of the normal swallowing process, dysphagia, safe swallowing strategies, swallowing assessment, importance of dysphagia screening, I-RBWH dysphagia screening tool structure and aims were provided. In order to assess the effectiveness of the training, a short 20 true/false questionnaire was provided before and after training. A pass rate of $80 \%$ in the total score of the questionnaire was set. Nurses who failed to meet these criteria underwent further training. All the eight nurses employed in the subacute care Unit of our hospital were enrolled.

\section{Reliability analysis (phase 3)}

Clinical data were obtained from 105 consecutive patients (49 men and 56 women). The mean age of participants was $76.7 \pm 7.5$ years (range 34-94). The mean age of female patients was $77.3 \pm 10.3$ years (range $34-94$ ), while male patients were aged $76.1 \pm 10.9$ years (range $43-93$ ). Clinical conditions leading to admission to the subacute care Unit are reported in Table I; the large majority of enrolled patients presented with comorbidities.

To assess intra-rater reliability of the I-RBWH dysphagia screening tool each patient was assessed twice, at admission to the Unit and within 24 hours, by the same nurse. This interval period was selected because no substantial change was expected to take place in subjects' deglutition abilities within this period. While completing the second I-RBWH dysphagia screening tool, nurses did not have any chance to check the results obtained during the first evaluation. To assess inter-rater reliability, each patient was also evaluated by a SLP specialised in swallowing disorders within 24 hours from admission. The SLP administered the I-RBWH dysphagia screening tool, but differently from the study of Cichero et al. ${ }^{2}$, the SLP was blind to the results obtained during nurses' administration.

\section{Screening accuracy (phase 4)}

As in the original study, for analysis of screening accuracy of the I-RBWH dysphagia screening tool, each patient was evaluated by a SLP using standardised clinical assessment of swallowing with the Mann assessment of swallowing ability (MASA), a dysphagia clinical assessment tool validated for stroke patients ${ }^{30}$. This latter scores 
oral motor/sensory features of swallowing, patient cooperation and comprehension, dietary recommendations and predictive risk rating of swallowing integrity. The SLP administered both the I-RBWH and the MASA at the same time. The results of MASA assessment were dichotomised to distinguish between patients with and without dysphagia ( $\leq 178$ and $>178$ points respectively). The MASA scores and the results of the I-RBWH dysphagia screening tool obtained during the nurse administration were compared to evaluate the sensitivity and specificity ratings and positive and negative predictive values of the I-RBWH dysphagia screening tool.

\section{Statistical analysis}

Statistical tests were performed using SPSS 21.0 statistical software (SPSS, Inc., Chicago, IL). The effect of nurse training was evaluated using Wilcoxon signed-ranks test. Differences in the age of male and female patients were evaluated using the Mann-Whitney test. Kappa coefficient was used to evaluate intra- and inter-rater reliability of IRBWH dysphagia screening tool. The screening accuracy was evaluated by assessing the sensitivity, specificity, positive predictive value and negative predictive value of the I-RBWH dysphagia screening tool by comparing its results with the MASA scores. For all comparisons, a $p$ value $<0.05$ was considered significant.

\section{Results}

As far as the feeding recommendation is concerned, of the 105 consecutive patients enrolled, at admission 98 were following a regular diet, 6 were fed through a nasogastric tube (NGT) and 1 had PEG in situ. No differences were found in the distribution of age between male and female patients on Mann-Whitney test $(\mathrm{p}=0.39)$.

Nurse training (phase 2)

A total of 8 nurses, with a bachelor university degree in nursing, were trained. The mean pre-training score of the 20 items questionnaire was $14.4 \pm 2.7$ (range 12-17) of a total possible score of 20 . The mean post-training score was $18.7 \pm 2.5$ (range 18-20). The improvement in the score obtained by nurses after the training was significant by Wilcoxon signed rank test $(Z=4.102 ; \mathrm{p}<0.001)$.

After the training session, none of the nurses failed the pass rate of $80 \%$ in the total score of the questionnaire.

\section{Reliability analysis (phase 3)}

All 105 patients were involved in reliability analysis. The first screening was administered by a nurse immediately at admission to the subacute care Unit. The same nurse managed to repeat the screening within 24 hours. The time between the first administration of the I-RBWH dysphagia screening tool and the SLP assessment was almost always within one to six hours. The compliance rate was
$100 \%$ for both nurses and the SLP. As far as the nurse's first I-RBWH dysphagia screening tool administration is concerned, 28 individuals (27\%) failed the screening and were consequently identified as "at risk" for dysphagia and aspiration. During the nurse's second administration of the I-RBWH screening tool, 27 patients failed the screening, while 78 passed it. The kappa coefficient demonstrated a significant agreement between test and re-test condition (kappa $=0.92 ; \mathrm{p}<0.001)$ thus demonstrating a strong intra-rater reliability.

As far as inter-rater reliability is concerned, the I-RBWH results obtained during SLP's and nurse's administration of the tool on the same patient were compared. The kappa coefficient demonstrated a significant agreement between nurse and SLP results of I-RBWH (kappa $=0.88$; $\mathrm{p}<0.001$ ), thus demonstrating a strong inter-rater reliability.

\section{Screening accuracy (phase 4)}

All 105 enrolled patients were screened by a total of 8 trained nurses at admission. The first phase of the I-RBWH dysphagia screening tool took approximately two minutes to complete, while 5-7 minutes were necessary to complete the second phase (which included questions specifically related to feeding and swallowing) and the water swallow test.

In Figure 1 the number of patients who passed or failed each phase of the I-RBWH dysphagia screening tool at first assessment is reported. Seventy-eight patients failed

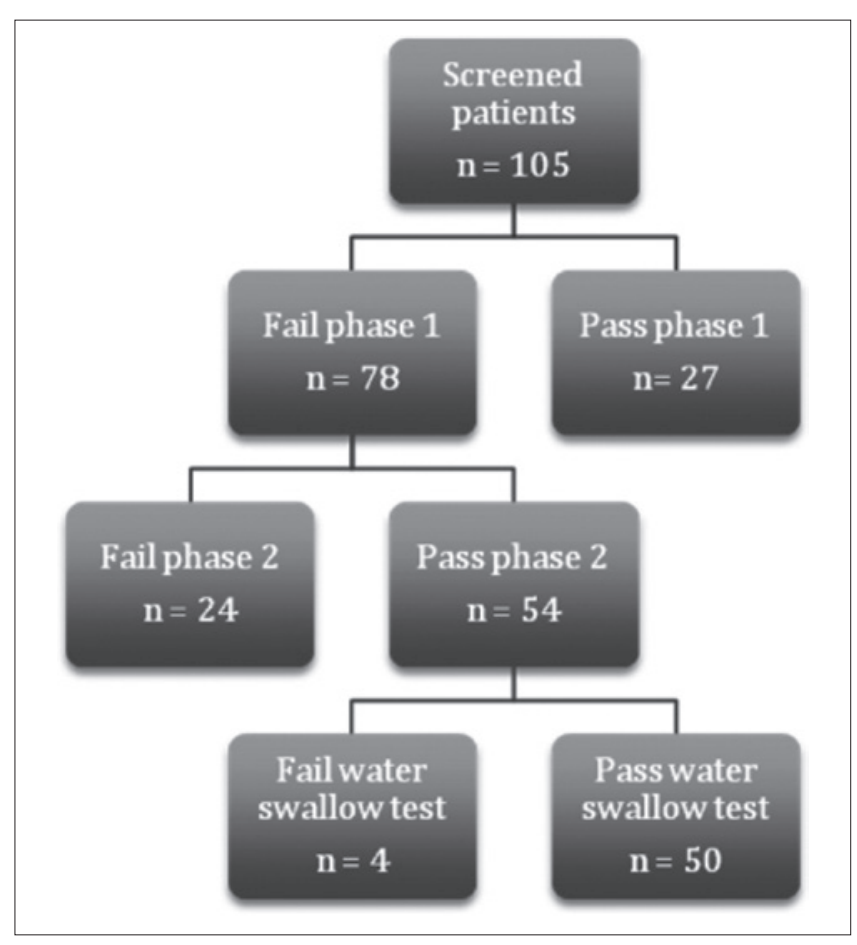

Fig. 1. Results of the first nurse administration of the I-RBWH dysphagia screening tool on 105 consecutive enrolled patients. 
phase 1 of the I-RBWH dysphagia screening tool; the underlying causes of failure are reported in Table II. Twenty-four of the 78 patients who failed phase 1 also failed phase 2 and were placed on NBM. The remaining54 patients who failed phase 1 underwent a water swallow test; four failed the water swallow test and were placed on NBM.

Results of MASA evaluation performed by SLP showed that a total of 78 patients scored above 178 points (mean $194.7 \pm 5.9$; range 179-205) and were consequently considered not dysphagic. Twenty-seven patients scored equal or lower than 178 points (mean $154.2 \pm 29.1$; range 77-178) and were considered dysphagic. In order to evaluate the sensitivity, specificity, positive and negative predictive values for the dysphagia screening tool, nurse screening results were compared to MASA scores. Sensitivity rating for the I-RBWH dysphagia screening tool was $93 \%$, while specificity was $96 \%$. The positive predictive value was $90 \%$, while the negative predictive value was $97 \%$ (Table III).

At the completion of the screen, nursing staff allocated 77 patients, who were found negative at I-RBWH screening, to an oral diet. Following SLP assessment, none of them was considered unsuitable for oral liquids. Fifty-six patients were confirmed on a general diet, while the remaining 21 required a modified texture diet because partially or fully edentulous. Twenty-eight patients failed the screening, were placed on NBM and referred to SLP: 21 required a modified texture diet, 6 were placed on NBM, while 1 was placed on regular diet (Table IV). Three of the 28 patients who failed the I-RBWH were not considered dysphagic at MASA: in one case the patient did not adequately manage oral secretions at nurse assessment, and were considered an appropriate over-referral; the remaining two cases were judging errors by nursing staff. Two of the 27 patients considered dysphagic at MASA passed the I-RBWH: in one case the nurse did not recognise the gurgly voice, and in the other case the nurse did not recognise the cough that occurred immediately after the swallow. In both cases, compensatory strategies by the SLP were sufficient to allow safe oral liquid administration.

\section{Discussion}

The RBWH dysphagia screening tool is unique since it uses diagnostic categories, patient self-report, nursing observation and - if applicable - the water swallow test. In the original study, high scores for sensitivity, specificity, positive and negative predictive values were found and the authors speculated that these results were related to the inclusion of all the above mentioned parameters in a single screening tool ${ }^{2}$. However, the lack of blinding between RBWH dysphagia screening tool scores and speech pathology clinical assessment of swallowing suggested caution in the interpretation of these results.
Table II. Number of patients considered at risk of dysphagia during first phase of the I-RBWH dysphagia screening tool administered by nurses.

\begin{tabular}{lc} 
Aetiology identified as a risk factor for dysphagia & $\begin{array}{c}\text { Number of } \\
\text { patients }\end{array}$ \\
Dysphagia or aspiration on previous admission(s) & 14 \\
Chronic obstructive pulmonary disease \pm upper & 9 \\
gastrointestinal disorder & 5 \\
Cerebrovascular accident (stroke) & 21 \\
Neurological involvement & 1 \\
Head injury & 1 \\
Head and neck surgery & 1 \\
Chemotherapy/radiation to head and neck & 18 \\
Acutely unwell, frail aged with co-morbidities & 6 \\
Suspected aspiration pneumonia/recurrent chest infections & 2 \\
Severe disability (e.g. physical disability) & 78 \\
Total & \\
\hline
\end{tabular}

Table III. Analysis of the I-RBWH dysphagia screening tool accuracy by comparing its results with MASA scores.

\begin{tabular}{lccc} 
& MASA positive & MASA negative & Total \\
RBWH fail & 25 & 3 & 28 \\
RBWH pass & 2 & 75 & 77 \\
Total & 27 & 78 & 105 \\
\hline
\end{tabular}

Table IV. Feeding recommendations after SLP blind evaluation.

\begin{tabular}{lcccc} 
& \multicolumn{2}{c}{ Pass I-RBWH } & \multicolumn{2}{c}{ Fail l-RBWH } \\
& $\mathbf{N}=\mathbf{7 7}$ & Percentage & $\mathbf{N}=\mathbf{2 8}$ & Percentage \\
Regular diet & 56 & $74 \%$ & 1 & $3 \%$ \\
Modified texture & 21 & $26 \%$ & 21 & $75 \%$ \\
NBM & 0 & $0 \%$ & 6 & $22 \%$ \\
\hline
\end{tabular}

In the present study, the psychometric properties of the I-RBWH dysphagia screening tool were studied. The results showed strong intra- and inter-rater reliability and good screening accuracy. These results are similar to those of Cichero et al. ${ }^{2}$ and further support the use of the I-RBWH screening tool in patients admitted to a subacute care unit. In addition, different from the original report, in the present study a blinding protocol for SLP evaluation was established, increasing confidence in the interpretation of the results.

Specific findings related to the I-RBWH dysphagia screening tool are noteworthy. In particular, the compliance rate was $100 \%$. This high compliance rate may suggest that the nurses fully understood the importance of screening for dysphagia and consequently were more motivated in administration of the screening tool. It is possible that the nurses training program played an important role since information regarding swallowing assessment and importance of dysphagia screening were provided. In addition, it is possible to speculate that the I-RBWH dysphagia 
screening tool is not a burdensome instrument and that it can be easily administered since the time required to complete the screening procedure never exceeded 9 minutes. Cichero et al. ${ }^{2}$ reported a compliance rate of $84 \%$. It is possible that the higher rate reported in the current study was related to the smaller number of nurses $(n=8)$ and hospital wards $(n=1)$ participating in the trial. In the original study, in fact, 2 general medical wards participated in the study, and a total of 38 nurses were trained.

As far as reliability of the I-RBWH dysphagia screening tool is concerned, both intra- and inter-rater reliability were analysed: the scores obtained support the idea that the I-RBWH has a high stability and reproducibility over time. In fact, the kappa coefficient for intra-rater reliability was 0.92 , while that for inter-rater reliability was 0.88 . No data regarding the reliability of the RBWH screening tool were provided in the original study ${ }^{2}$.

In the current study, the sensitivity and specificity ratings for the I-RBWH were $93 \%$ and $96 \%$ respectively, while the positive predictive and negative predictive values were $90 \%$ and $97 \%$, respectively, when the I-RBWH dysphagia screening tool scores were compared with MASA examination. These results appear slightly lower than those reported in the study of Cichero et al. ${ }^{2}$. However, it is possible that these differences are related to the lack of blinding of the original study. The SLPs who performed the MASA examination in the original study, in fact, knew the results of RBWH dysphagia screening tool.

Twenty-eight of the screened patients $(26.67 \%)$ presented with dysphagia. This data is in agreement with previous reports ${ }^{231-33}$. In particular, Cichero et al. ${ }^{2}$ reported that $25 \%$ of patients were positive at RBWH screening, while the estimated incidence of dysphagia in hospitalised patients ranges between $15 \%$ and $30 \%{ }^{13132}$. The high rate of dysphagia in the present study supports the need for a valid dysphagia screening tool in subacute care settings. A formal dysphagia screening tool, in fact, may reduce the risk of a patient starting oral intake inappropriately or unsafely. For this purpose, the I-RBWH dysphagia screening tool appears optimal since it demonstrated strong reliability and good accuracy. In addition, it is designed to be administered by nursing staff (present 24 hours a day, 7 days a week) and allows rapid patient identification and timely referral for ongoing care. The prevention of clinical complications related to dysphagia, such as malnutrition, dehydration and aspiration pneumonia ${ }^{532}$, may reduce mortality and recovery time in hospital ${ }^{2}$ and may also reduce healthcare costs. The findings of the present study support the application of the triaging concept; only 54 patients of the 105 $(51.42 \%)$ required a water swallow test, while the remaining 51 patients $(48.58 \%)$ were screened on the basis of phase 1 (information from clinical records) and 2 data (information from clinical records or from patients or caregivers). All the patients, except 1, who were referred to the SLP required a modified diet.
There are several limitations in the study. First, the study population included only 105 patients; therefore, although the data herein encourage the use of the I-RBWH, they should be considered preliminary. Second, the study included patients from a single subacute care unit; thus, it is unknown how these findings could be generalised to subacute care unit in general. Third, the accuracy of the I-RBWH screening tool was not tested against an instrumental assessment (such as FEES or VFSS); future studies are needed to further analyse the sensitivity and specificity of the I-RBWH.

\section{Conclusions}

In conclusion, our current findings support the reliability and screening accuracy of the I-RBWH dysphagia screening tool for the screening of patients in subacute care settings. The application of the I-RBWH dysphagia screening tool in daily clinical practice as well as in epidemiological, efficacy and outcome studies is recommended.

\section{References}

Ferraris VA, Ferraris SP, Moritz D, et al. Oropharyngeal dysphagia after cardiac operations. Ann Thor Surg 2001;71: 1792-6.

2 Cichero JAY, Heaton S, Bassett L. Triaging dysphagia: nurse screening for dysphagia in an acute hospital. J Clin Nurs 2009; 18:1649-59.

3 Lin LC, Wu SC, Chen WS, et al. Prevalence of impaired swallowing in institutionalized older people in Taiwan. J Am Geriatr Soc 2002;50:1118-23.

4 Coates C, Bakheit AM. Dysphagia in Parkinson's disease. Eur Neurol 1997;38:49-52.

5 Stratton, RJ, Green CJ, Elia M. Disease-related malnutrition: an evidence-based approach to treatment. CABI, Wallingford 2003.

6 Odderson IR, Keaton JC, McKenna BS. Swallow management in patients on an acute stroke pathway: quality is cost effective. Arch Phys Med Rehab 1995;76:1130-3.

7 Hinchey JA, Shephard T, Furie K, et al. Stroke practice improvement investigators. Formal dysphagia screening protocols prevent pneumonia. Stroke 2005;36:1972-6.

8 Dennis MS, Lewis SC, Warlow C. FOOD Trial Collaboration. Effect of timing and method of enteral tube feeding for dysphagic stroke patients (FOOD): a multicentre randomised controlled trial. Lancet 2005;365:764-72.

9 Carnaby G, Hankey GJ, Pizzi J. Behavioural intervention for dysphagia in acute stroke: a randomized trial. Lancet Neurol 2006;5:31-7.

10 Aviv JE, Thomson JE, Sunshine S, et al. Fiberoptic endoscopic evaluation of swallowing with sensory testing (FEESST) in healthy controls. Dysphagia 1998;13:87-92.

11 Logemann JA. Rehabilitation of oropharyngeal swallowing disorders. Acta Otorhinolaryngol Belg 1994;48:207-15.

12 Farneti D, Fattori B, Nacci A, et al. The Pooling-score ( $P$ score): inter- and intra-rater reliability in endoscopic assess- 
ment of the severity of dysphagia. Acta Otorhinolaryngol Ital 2014;34:105-10.

13 Daniels SK, Schroeder MF, DeGeorge PC, et al. Defining and measuring dysphagia following stroke. Am J Speech Lang Pathol 2009;18:74-81.

${ }^{14}$ Ramsey DJ, Smithard DG, Kalra L. Early assessments of dysphagia and aspiration risk in acute stroke patients. Stroke 2003;34:1252-7.

15 DePippo KL, Holas MA,Reding MJ. The Burke dysphagia screening test: validation of its use in patients with stroke. Arch Phys Med Rehabil 1994;75:1284-6.

16 Ellul J, Barer D. on behalf of ESDB/COSTAR Collaborative Dysphagia Study Interobserver reliability of a Standardised Swallowing Assessment (SSA). Cerebrovascular Dis 1996;2:152-3.

17 Logemann JA, Veis S, Colangelo L. A screening procedure for oropharyngeal dysphagia. Dysphagia 1999;14:4451.

18 Perry L, Love CP. Screening for dysphagia and aspiration in acute stroke: a systematic review. Dysphagia 2001;16:7-18.

19 Perry L. Screening swallowing function of patients with acute stroke. Part one: Identification, implementation and initial evaluation of a screening tool for use by nurses. J Clin Nurs 2001;10:463-73.

20 Stewart L. Development of the nutrition and swallowing checklist, a screening tool for nutrition risk and swallowing risk in people with intellectual disability. J Int Dev Dis 2003;28:171-87.

21 Westergren A. Detection of eating difficulties after stroke: a systematic review. Int Nurs Rev 2006;53:143-9.

${ }^{22}$ Martino R, Silver F, Teasell R, et al. The Toronto Bedside Swallowing Screening Test (TOR-BSST): development and validation of a dysphagia screening tool for patients with stroke. Stroke 2009;40:555-61.

23 Rofes L, Arreola V, Clavé P. The volume-viscosity swallow test for clinical screening of dysphagia and aspiration. Nestle Nutr Inst Workshop Ser 2012;72:33-42.

24 Schindler A, Vincon E, Grosso E, et al. Rehabilitative management of oropharyngeal dysphagia in acute care settings: data from a large Italian teaching hospital. Dysphagia 2008;23:230-6

25 Suiter DM, Sloggy J, Leder SB. Validation of the Yale Swallow Protocol: a prospective double-blinded videofluoroscopic study. Dysphagia 2014;29:199-203.

26 Cochrane AL, Holland WW. Validation of screening procedures. Br Med Bull 1971;27:3-8.

27 Trapl M, Enderle P, Nowotny N, et al. Dysphagia bedside screening for acute-stroke patients. The gugging swallowing screen. Stroke 2007;38:1-7.

28 Beaton DE, Bombardier C, Guillemin F, et al. Guidelines for the process of cross-cultural adaptation of self-report measures. Spine 2000;25:3186-91.

29 Boynton PM, Greenhalgh T. Selecting, designing, and developing your questionnaire. BMJ 2004;328:1312-15.

30 Mann GA. MASA: the Mann Assessment of Swallowing Ability. Clifton Park, NY: Singular, Thomson Learning 2002.

31 Barczi SR, Sullivan PA, Robbins J. How should dysphagia care of older adults differ? Establishing optimal practice patterns. Sem Speech Lang 2000;21:347-61.

32 Foley N, Martin RE, Salter KL, et al. A review of the relationship between dysphagia and malnutrition following stroke. $\mathrm{J}$ Rehabil Med 2009;41:707-13.

33 Groher ME, Bukatman R. The prevalence of swallowing disorders in two teaching hospitals. Dysphagia 1986;1:3-6. 